\title{
Propensity-matched comparison of balloon-expandable and self- expanding valves for transcatheter aortic valve replacement in a Chinese population
}

\author{
Wen-Bin Ou-Yang ${ }^{1}$, Wei Wang ${ }^{1}$, Jie Dong ${ }^{1}$, Yong-Quan Xie ${ }^{1}$, Jun-Yi Wan ${ }^{1}$, Zi-Qi Yue ${ }^{1}$, \\ Shou-Zheng Wang ${ }^{1}$, Hong Meng ${ }^{2}$, Xu Wang ${ }^{1}$, Dong-Hui Xu ${ }^{1}$, Feng-Wen Zhang ${ }^{1}$, Jing Dong ${ }^{1}$, \\ Xiang-Bin Pan ${ }^{1}$, Ge-Jun Zhang ${ }^{1}$ \\ ${ }^{1}$ Department of Structural Heart Disease, National Center for Cardiovascular Disease, China \& Fuwai Hospital, Chinese Academy of Medical \\ Sciences \& Peking Union Medical College, Beijing, China; ${ }^{2}$ Department of Echocardiography, National Center for Cardiovascular Disease, China \& \\ Fuwai Hospital, Chinese Academy of Medical Sciences \& Peking Union Medical College, Beijing, China \\ Contributions: (I) Conception and design: XB Pan, GJ Zhang, WB Ou-Yang; (II) Administrative support: XB Pan, H Meng; (III) Provision of \\ study materials or patients: XB Pan, W Wang, GJ Zhang; (IV) Collection and assembly of data: YQ Xie, JY Wan, ZQ Yue, H Meng, J Dong; (V) \\ Data analysis and interpretation: SZ Wang, FW Zhang, J Dong, X Wang, DH Xu; (VI) Manuscript writing: All authors; (VII) Final approval of \\ manuscript: All authors. \\ Correspondence to: Xiang-Bin Pan; Ge-Jun Zhang. Department of Structural Heart Disease, Fuwai Hospital, 167 Beilishi Road, Xicheng District, \\ Beijing 100037, China. Email: panxiangbin@fuwaihospital.org; gjzhang212@163.com.
}

Background: Balloon-expandable valves (BEV) and self-expanding valves (SEV) for transcatheter aortic valve replacement (TAVR) have shown promising results in Western populations. Herein, we comparatively evaluated their hemodynamics and early clinical outcomes in a Chinese population.

Methods: One hundred seventy-eight patients with symptomatic aortic stenosis who had undergone transfemoral TAVR using SEV ( $\mathrm{n}=153$; Venus-A, 97; VitaFlow, 56) or BEV ( $\mathrm{n}=25$; Sapien3) from September 2020 to April 2021 were retrospectively enrolled, and 25 pairs were propensity-score matched for 10 baseline variables. The primary study outcomes were aortic valve hemodynamics and postoperative complications at discharge and 3-month follow-up.

Results: TAVR was successful in all patients. Compared with SEV group, the BEV group had similarly distributed baseline characteristics, procedural time, hospital stay, new pacemaker implantation, and paravalvular regurgitation grade. We also observed that the BEV group had lower rates of balloon predilation (60\% vs. 92\%, $\mathrm{P}=0.018)$, post-dilation ( 0 vs. 20\%, $\mathrm{P}=0.050)$ and second valve implantation ( 0 vs. $24 \%, \mathrm{P}=0.022)$; higher mean transaortic gradient $(14.3 \pm 6.1$ vs. $10.8 \pm 4.9, \mathrm{P}=0.030)$ and proportion of patients with elevated gradients $(20 \%$ vs. $0, \mathrm{P}=0.050)$ at discharge; and similar rehospitalization, mean transaortic gradient, new pacemaker implantation, and paravalvular regurgitation grade than the SEV group at the 3-month follow-up. There were no deaths in either group. However, the proportion of patients with elevated gradients in SEV group was higher at 3 months than before discharge (24\% vs. 0, $\mathrm{P}=0.022$ ).

Conclusions: BEV and SEV for transfemoral TAVR appear comparably safe and effective, with high device success and favorable 3 -month clinical outcomes. However, the transaortic gradient and new pacemaker implantation in the SEV group increased during follow-up, warranting larger studies with longerterm follow-up.

Keywords: Transcatheter aortic valve replacement (TAVR); propensity-matched analysis; self-expanding valves; balloon-expandable valves

Submitted Nov 08, 2021. Accepted for publication Jan 19, 2022.

doi: $10.21037 /$ atm-21-6637

View this article at: https://dx.doi.org/10.21037/atm-21-6637 


\section{Introduction}

Introduced in 2002 (1), transcatheter aortic valve replacement (TAVR) has since become an effective alternative to surgical aortic valve replacement in lowto-high-risk patients with symptomatic aortic stenosis (2-6). The two commercially available design categories of transcatheter heart valves (THV), namely balloonexpandable valves (BEV) and self-expanding valves (SEV), each have their own advantages and disadvantages. Previous studies have directly compared the efficacy and safety of these devices in Western (7-9) but not Chinese populations, partly because the new generation Sapien ${ }^{\mathrm{TM}}$ (Sapien3, Edwards Lifesciences, Irvine, CA) BEV became available in China in September 2020.

Relative to Western TAVR candidates, Chinese patients have a higher proportion of bicuspid aortic valve and degree of aortic valve calcification, more often aortic regurgitation than stenosis, and a smaller femoral artery diameter (10-13); however, both valve types were empirically recommended for use based on cardiac team experience in most clinical situations. Therefore, the present study evaluated the valve hemodynamics and early clinical outcomes of SEV (including Venus-A, Venus MedTech, Hangzhou, China; VitaFlow, MicroPort ${ }^{\circledR}$, Shanghai, China) and BEV (Sapien3, the only BEV applied in China) using a propensity-matched comparison approach. We present the following article in accordance with the STROBE reporting checklist (available at https://atm.amegroups.com/article/view/10.21037/atm$21-6637 / \mathrm{rc})$.

\section{Methods}

\section{Patients}

This retrospective, observational, cohort study analyzed data collected from consecutive patients with symptomatic, severe aortic stenosis who underwent transfemoral TAVR between September 2020 and April 2021. The procedure was completed by three TAVR qualified teams at the Structural Heart Disease Center of Fuwai Hospital. Patients with pure aortic regurgitation or missing baseline data necessary for the propensity score were excluded. The study was approved by the ethics board of Fuwai Hospital, Chinese Academy of Medical Sciences (No. 2021-1637). All patients and legal guardians had signed informed consent for the procedure and clinical record review. The study complied with the Declaration of Helsinki (as revised in 2013) and all relevant Chinese laws.
Pre-procedure baseline assessment included clinical data (patient demographics, symptoms, comorbidities, routine laboratory testing, and risk evaluation), electrocardiogram, transthoracic echocardiography, and cardiac computed tomography angiography (CTA). All TAVR candidates were evaluated by the local multidisciplinary heart teams and core laboratory. The THV type and size were chosen based on the experience and preferences of each team.

\section{Procedure description}

The prosthetic THVs used in this study (Sapien3, Venus-A, and VitaFlow) have previously been described in detail (14-16). Procedures were performed under conscious sedation or general anesthesia in the hybrid operating room or catheterization laboratory. The right femoral artery was used for valve-stent delivery, and the left femoral artery was punctured for coronary angiography. A temporary pacing lead was inserted through the right internal jugular vein. Standard THV implantation techniques were used according to the size and morphological characteristics of the valve (14-16). Postprocedural events were evaluated by angiocardiography and transthoracic echocardiography.

\section{Data definitions}

Baseline and outcome data were obtained through a detailed chart review of each patient. Paravalvular and aortic regurgitation grade were defined as 0 if absent, 1 if trivial, 2 if mild, 3 if moderate, and 4 if severe by transthoracic echocardiography (17). The aortic valve gradient was measured by transthoracic echocardiography. The prosthetic valve diameter index was defined as the diameter of the implanted valve by cardiac CTA indexed to body surface area.

\section{Follow-up}

Follow-up assessments of clinical outcomes, transthoracic echocardiography, and electrocardiography were conducted via outpatient visits or phone interviews at $1,3,6$, and 12 months, and adverse events and prosthetic valve hemodynamics were recorded. The last follow-up was on July 31, 2021.

\section{Statistical analysis}

Continuous variables were expressed as means \pm standard 


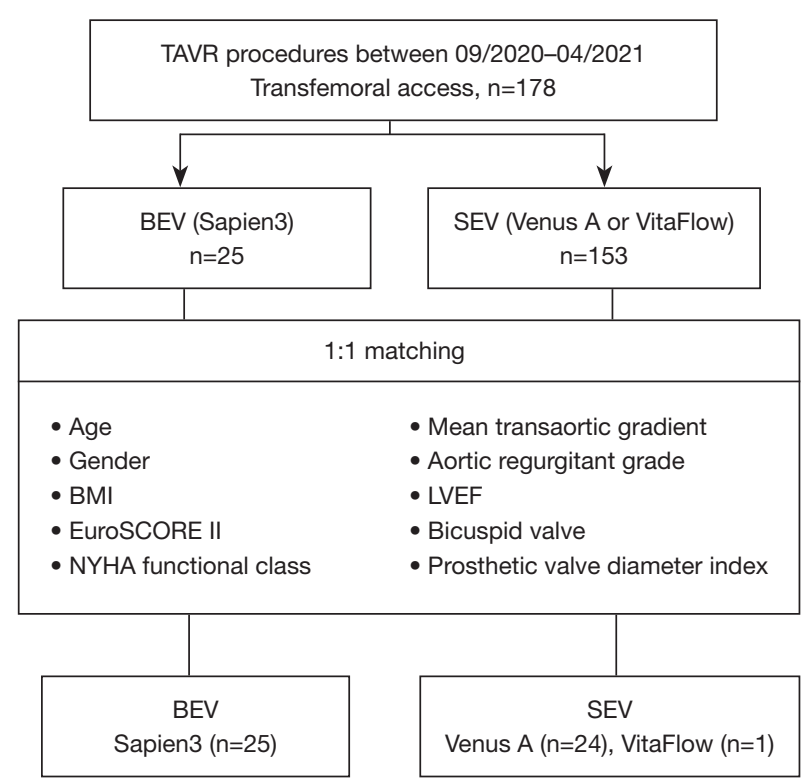

Figure 1 Study flow and variables used for propensity matching. TAVR, transcatheter aortic valve replacement; BEV, balloonexpandable valves; SEV, self-expanding valves; BMI, body mass index; NYHA, New York Heart Association; LVEF, left ventricular ejection fraction.

deviations or medians with interquartile ranges as appropriate and were compared using $t$-test or nonparametric test as appropriate. Meanwhile, categorical variables were expressed as frequencies and percentages and were compared using the Chi-square test or the Fisher's exact test as appropriate. SPSS 25.0 for Windows (SPSS Inc., Chicago, USA) was used for statistical analysis. A twosided $\mathrm{P}$ value of less than 0.05 was considered statistically significant.

Due to the large difference in the amount of data between BEV and SEV, propensity score matching was applied to adjust the influence of selection bias and potential confounders (Figure 1). Propensity scores were calculated using a non-parsimonious multivariate logistic regression model with 10 variables, including age, gender, body mass index, bicuspid valve, EuroSCORE II, New York Heart Association (NYHA) functional class, left ventricular ejection fraction (LVEF), aortic regurgitant grade, mean transaortic gradient, and prosthetic valve diameter index. Patients in the BEV and SEV groups were matched at a 1:1 ratio using the nearest neighbor matching algorithm. The criterion for matching pairs used a caliper width equal to 0.2 of the pooled standard deviation of the logit of propensity score. Finally, 25 pairs of patients were matched with similarly distributed baseline data between the two groups.

\section{Results}

\section{Baseline characteristics (Table 1)}

Among the 178 patients with symptomatic aortic stenosis who had undergone TAVR from September 2020 to April 2021 by three TAVR qualified teams at Fuwai Hospital, SEV (Venus-A, 97; VitaFlow, 56) was used in 153 patients and BEV (Sapien3) was utilized in 25 patients. There were no significant between-group differences in age, gender, body mass index, comorbidities, mean transaortic gradient, and proportion of bicuspid valve, among other variables. However, compared with BEV group, patients in SEV group had a lower LVEF $(56.6 \pm 13.1$ vs. $62.2 \pm 10.6, \mathrm{P}=0.024)$ and larger implanted valve size (26 vs. $23, \mathrm{P}=0.014)$ before matching.

After 1:1 propensity score matching for the variables summarized in Figure 1, 25 patients receiving BEV were well matched with 25 patients receiving SEV (Venus-A, 24; VitaFlow, 1), without significant baseline differences (Table 1). The important factors that affect long-term survival and quality of life of patients after TAVR include preoperative severe comorbidities and postoperative adverse events. In particular, LVEF and implanted valve size, which directly affect procedural outcomes, were well balanced after matching.

\section{Intraprocedural data and pre-discharge results (Table 2)}

There was no stroke, life-threatening bleeding, conversion to surgery, or death in either group. The hospital course was uneventful, and both groups had a similar procedural time $(85.8 \pm 31.9$ vs. $100.7 \pm 38.8, \mathrm{P}=0.145)$, postprocedural hospital stay $(4.9 \pm 1.7 v$ s. $5.5 \pm 2.7, \mathrm{P}=0.354)$, new permanent pacemaker implantation (PPI:4\% vs. $8 \%, \mathrm{P}=1.000$ ), and paravalvular regurgitation (PVR) grade $(1$ vs. $1, \mathrm{P}=0.925$, Figure $2 A$ ). The rates of balloon pre- and post-dilation were significantly lower in the BEV group than the SEV group (pre-dilation: $60 \%$ vs. $92 \%, \mathrm{P}=0.018$; post-dilation: 0 vs. $20 \%, \mathrm{P}=0.050)$. No patients suffered valve displacement in the BEV group, and only one valve was implanted in each patient. However, a second valve was implanted in six patients (24\%) in the SEV group, including four patients with valve displacement and two patients with at least moderate PVR. The postprocedural mean transaortic 
Table 1 Baseline patient characteristics before and after propensity score matching

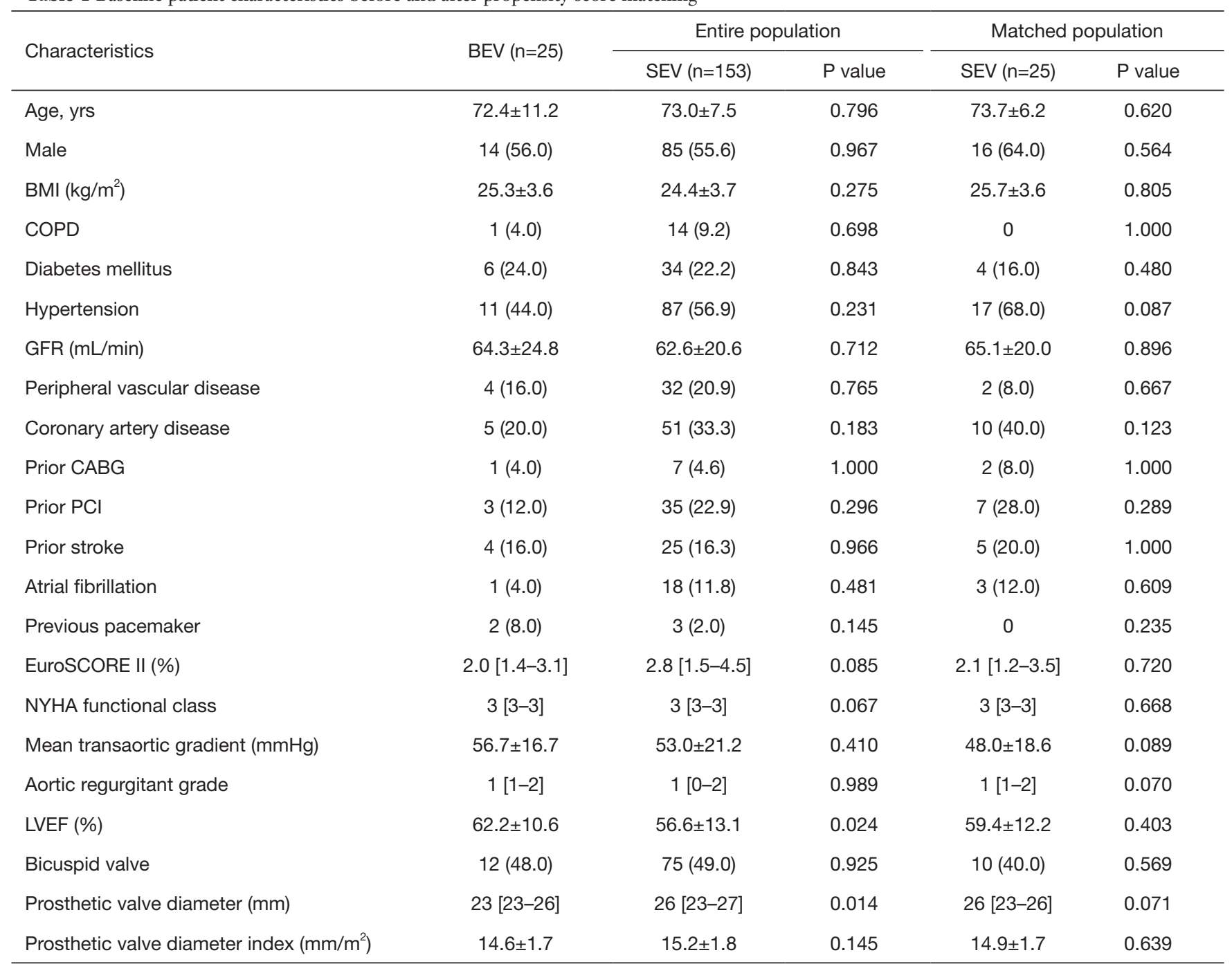

Data are presented as $\mathrm{n}(\%)$ or median [interquartile range] or mean \pm standard deviations. Regurgitant grade 0: absent, 1: trivial, 2: mild, 3: moderate, and 4: severe. BEV, balloon-expandable valves; SEV, self-expanding valves; BMI, body mass index; COPD, chronic obstructive pulmonary disease; GFR, glomerular filtration rate; CABG, coronary artery bypass grafting; PCI, percutaneous coronary intervention; NYHA, New York Heart Association; LVEF, left ventricular ejection fraction.

gradient was slightly higher in the BEV group compared to the SEV group $(14.3 \pm 6.1$ vs. $10.8 \pm 4.9, \mathrm{P}=0.030)$, and the proportion of patients with elevated gradients $(\geq 20 \mathrm{mmHg})$ was also higher in the $\mathrm{BEV}$ group $(20 \%$ vs. $0 \%, \mathrm{P}=0.050)$.

\section{Three-month follow-up outcomes (Table 3)}

All patients were followed up for 3 months, and there were no stroke, life-threatening bleeding, or death events. Patients in the BEV and SEV groups showed similar LVEF (62.1 \pm 5.2 vs. $58.2 \pm 9.2, \mathrm{P}=0.067)$, PVR grade (1 vs. 1 ,
$\mathrm{P}=0.381$, Figure $2 B)$, and mean transaortic gradient $(15.6 \pm 7.2$ vs. $12.5 \pm 6.8, \mathrm{P}=0.122)$, although they all were slightly higher than at discharge. Interestingly, at 3 months $v s$. before discharge, the proportion of patients with elevated gradients did not change significantly in the $\mathrm{BEV}$ group (20\% vs. $16 \%, \mathrm{P}=1.000)$, but increased significantly in the SEV group (0\% vs. $24 \%, \mathrm{P}=0.022)$.

Two patients in the BEV group and three patients in the SEV group were re-admitted to the hospital. One case in each group was readmitted due to cardiac insufficiency, and discharged after anti-heart failure treatment, while 
Table 2 Intraprocedural data and pre-discharge results of the two matched groups

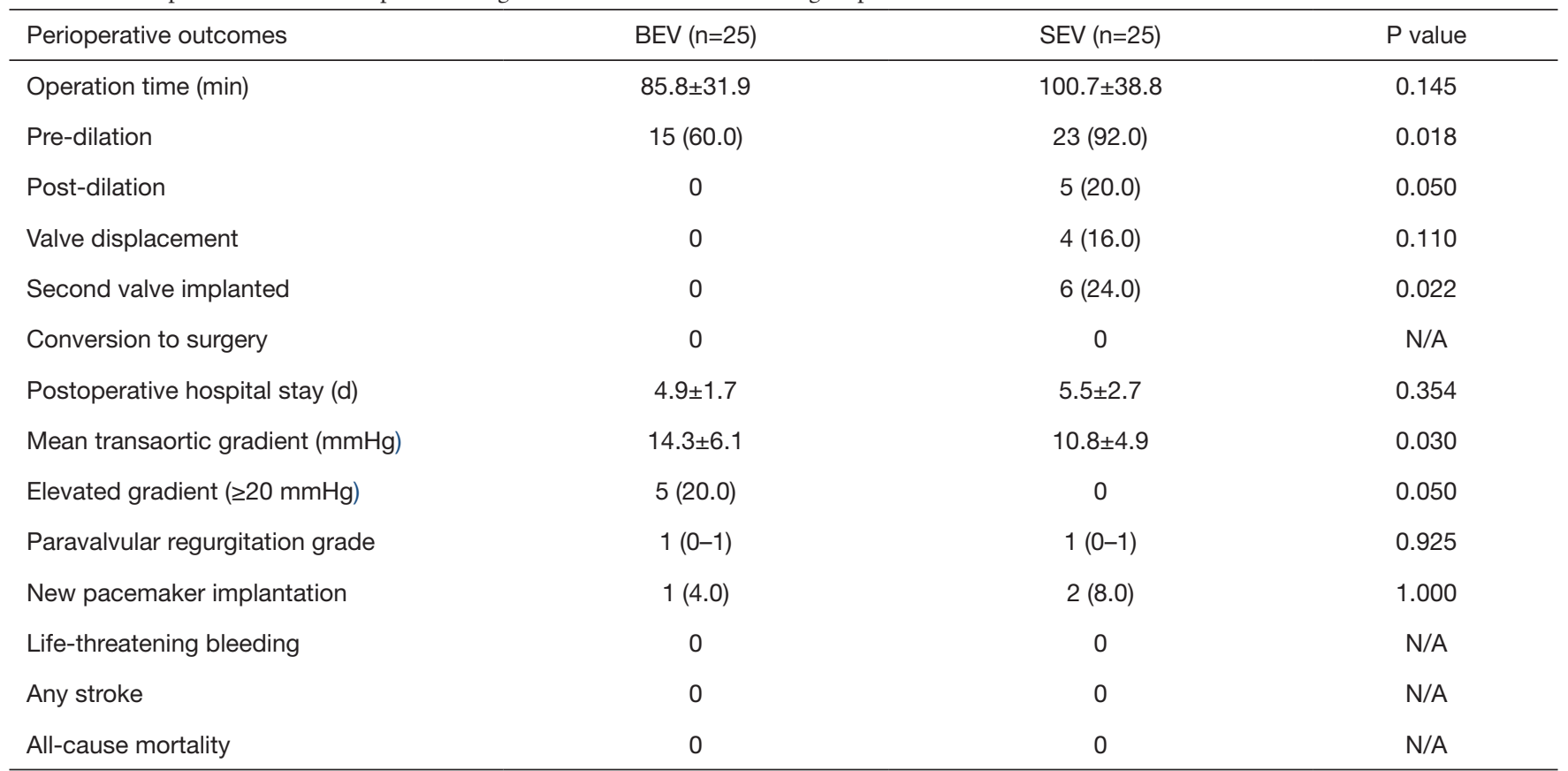

Data are presented as $\mathrm{n}(\%)$ or median (interquartile range) or mean \pm standard deviations. Paravalvular regurgitation grade 0: absent, 1 : trivial, 2: mild, 3: moderate, and 4: severe. BEV, balloon-expandable valves; SEV, self-expanding valves; N/A, not applicable.
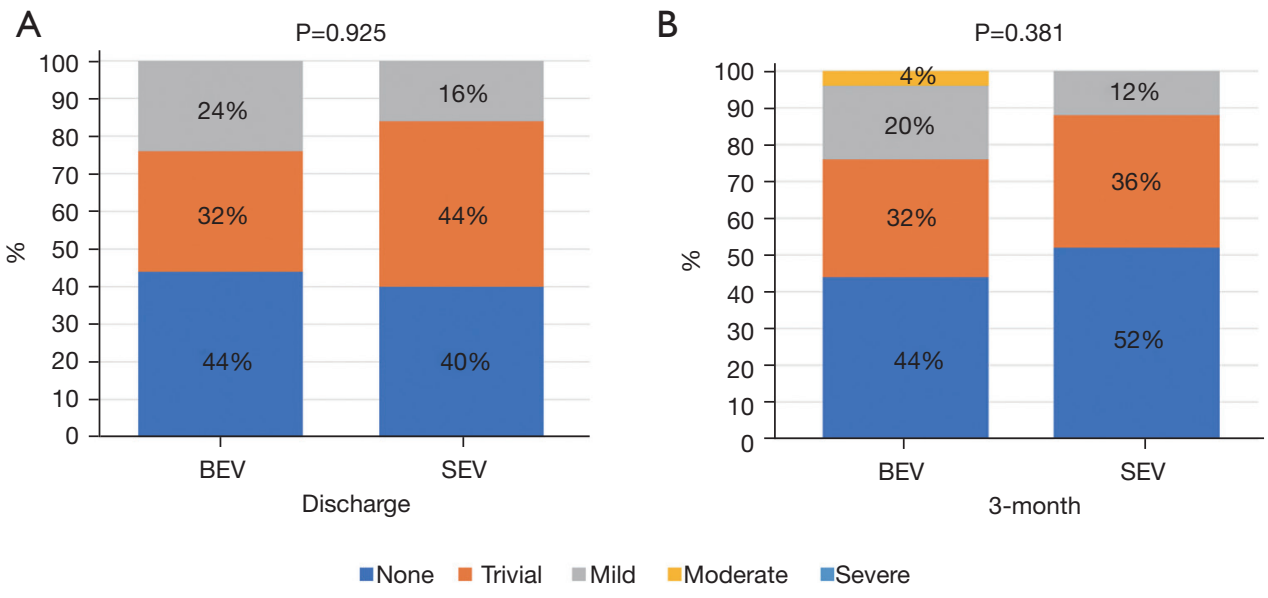

Figure 2 Paravalvular regurgitation (PVR). The incidence of PVR was comparable between the valves at discharge (A) and at the 3-month follow-up (B). BEV, balloon-expandable valves; SEV, self-expanding valves.

the remaining patients were readmitted for complete atrioventricular block with new PPI.

\section{Discussion}

The present study represents the first propensity-matched comparison of valve hemodynamics and early clinical outcomes between the new-generation BEV (Sapien3) and SEV (mostly Venus-A) for transfemoral TAVR in a Chinese population. The main findings of the study are as follows. Firstly, transfemoral TAVR using either valve system showed comparable safety and effectiveness with high device success and favorable 3-month clinical outcomes. Secondly, PVR grade and mean transaortic gradient grade 
Table 3 Clinical outcomes at the 3-month follow-up of the two matched groups

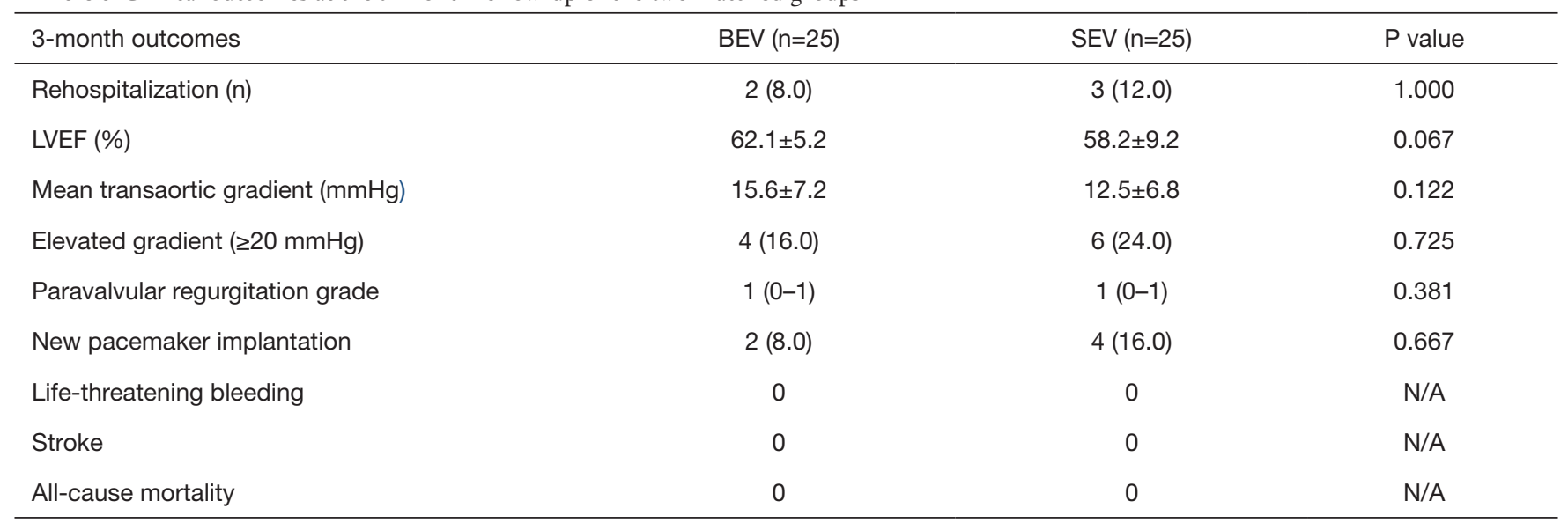

Data are presented as $\mathrm{n}(\%)$ or median (interquartile range) or mean \pm standard deviations. Paravalvular regurgitation grade 0: absent, 1: trivial, 2: mild, 3: moderate, and 4: severe. BEV, balloon-expandable valves; SEV, self-expanding valves; LVEF, left ventricular ejection fraction; N/A, not applicable.

were comparable between the groups, but the SEV group had higher rates of balloon pre-dilation, post-dilation, and second valve implantation. Thirdly, although the rate of new PPI was statistically similar between the groups, the proportion of patients with new PPI was double in the SEV group relative to the $\mathrm{BEV}$ group.

Due to anatomic challenges and high PVR, bicuspid valve anatomy was a relative contraindication to TAVR. Although a bicuspid aortic valve is present in $30 \%$ of patients undergoing aortic valve surgery (18), previous study has shown that bicuspid aortic stenosis accounts for only $3.3 \%$ of patients undergoing TAVR, representing a select group with more favorable anatomy (19). In our realworld study, although the prevalence of bicuspid anatomy approached $50 \%$, device implantation was successful in all cases without serious complications in either group, which was mainly due to the accurate preprocedural assessment by the core laboratory, continuous cumulative team experience, and improvement of the valve and delivery system design.

Excellent hemodynamics performance without elevated transvalvular gradient is crucial for the durability of prostheses (20). Some studies have demonstrated substantially different hemodynamic parameters between valve systems with significantly lower transvalvular gradients in SEVs at discharge and follow-up (20-22). The superior hemodynamic performance of SEVs is mainly based on its supra-annular valve design, while the intraannular valve design of BEV and the sealing skirt in the left ventricular outflow tract were responsible for the increase in transvalvular gradient. However, all studies compared the transvalvular gradient under different prosthetic valve diameter indexes. Therefore, we included the prosthetic valve diameter index among the matching variables. After matching, although the mean transaortic gradient and proportion of patients with elevated gradients were slightly higher before discharge in the BEV group than in the SEV group, hemodynamic performance was statistically similar between the groups at 3-month follow-up. Although the proportion of patients with elevated gradients did not change significantly in the BEV group, it increased significantly in the SEV group at 3-month follow-up relative to before discharge, which may be secondary to valve recoil, valve thrombosis, valve under expansion, or a chance finding (19). Therefore, postprocedural transaortic gradient in the SEV group warrants further study.

The presence of at least moderate PVR after TAVR has been associated with poorer prognosis $(23,24)$. Subgroups of TAVR patients, such as those with left ventricular hypertrophy, reduced ejection fraction, or additional comorbidities, may be affected by even mild PVR (25). The predictors of PVR include peri-annular calcium chunks or left ventricular outflow tract calcification, total extent of aortic valve calcification, and prosthesis undersizing and malpositioning (26). Therefore, to reduce PVR, Sapien3 was designed with a polyethylene terephthalate skirt that covers the lower portion of the frame, and delivery systems were optimized in both new-generation valves to minimize procedural difficulty and valve malpositioning. Recent 
studies have shown a low incidence of at least moderate PVR in about $1.8-3.4 \%$ of Sapien 3 patients $(7,21)$ and approximately $0-1.4 \%$ of SEV patients (Evolut, Evolut PRO, and VitaFlow) $(7,16)$. In our study, no patients had at least moderate PVR in either group before discharge. At 3 months, there was a case of at least moderate PVR in the BEV group (4\%), while PVR frequency in the SEV group tended to decline, which may be related to more frequent use of post-dilation and second valve implantation in the SEV group. In addition, SEV expands slowly after procedure, until finally fitting more closely with the autologous valve structure.

New PPI due to conduction abnormalities after TAVR was a common complication with varying incidence based on pre- and intra-procedural factors (27), ranging between $4 \%$ and $24 \%$ with BEV and $14.7 \%$ and $26.7 \%$ with SEV (even with new-generation devices) (28). Two studies have reported that the rate of new PPI was significantly higher with SEV compared to BEV $(27,29)$. Consistent with previous studies, our study showed a low rate of new PPI after using self-expanding Venus A (8\%), which was higher than that for balloon-expandable Sapien3 (4\%) before discharge. However, the incidence of new PPI doubled in both groups at the 3-month follow-up. Due to an apparent increase in mortality with new onset left bundle branch block and high atrioventricular block among patients undergoing TAVR (27), heart rhythm should be monitored regularly after discharge.

\section{Study limitations}

Despite its propensity-matched design and similarly distributed baseline characteristics between the study groups, interpretation of the findings of the present study is limited by its retrospective non-randomized design with a relatively small sample size and short follow-up duration. This is mainly due to the recent availability of the new generation balloon-expandable valves (Sapien3) in China. Therefore, a multi-center randomized trial with longerterm follow-up is warranted to compare both THVs. In addition, bias may have been introduced by the fact that the three teams performing TAVR had different professional backgrounds. Finally, the lack of transesophageal echocardiographic results is an important limitation of this study. Real-time three-dimensional transesophageal echocardiography is considered the most promising technique for quantitative assessment of heart valve disease. It can accurately measure parameters of the aortic root and left ventricular outflow tract, which can minimize the risk of clinically significant patient prosthesis mismatches and differentiate normal prosthetic valve function versus patient prosthesis mismatches versus acquired prosthetic valve stenosis.

\section{Conclusions}

Transfemoral TAVR using either BEV or SEV appears comparably safe and effective, with high device success and favorable 3-month clinical outcomes. However, the transaortic gradient and new PPI in the SEV group tended to increase during follow-up. Adequately powered larger studies with longer-term follow-up are needed to evaluate the benefit of each valve.

\section{Acknowledgments}

Funding: This work was supported by the Sanming Project of Medicine in Shenzhen (SZSM202011013), the National High Level Talents Special Support Plan (2020-RSW02), and CAMS Innovation Fund for Medical Sciences (2021I2M-1-065).

\section{Footnote}

Reporting Checklist: The authors have completed the STROBE reporting checklist. Available at https://atm. amegroups.com/article/view/10.21037/atm-21-6637/rc

Data Sharing Statement: Available at https://atm.amegroups. com/article/view/10.21037/atm-21-6637/dss

Conflicts of Interest: All authors have completed the ICMJE uniform disclosure form (available at https://atm. amegroups.com/article/view/10.21037/atm-21-6637/coif). The authors have no conflicts of interest to declare.

Ethical Statement: The authors are accountable for all aspects of the work in ensuring that questions related to the accuracy or integrity of any part of the work are appropriately investigated and resolved. The study was approved by the ethics board of Fuwai Hospital, Chinese Academy of Medical Sciences (No. 2021-1637). All patients and legal guardians had signed informed consent for the procedure and clinical record review. The study complied with the Declaration of Helsinki (as revised in 2013) and all relevant Chinese laws. 
Open Access Statement: This is an Open Access article distributed in accordance with the Creative Commons Attribution-NonCommercial-NoDerivs 4.0 International License (CC BY-NC-ND 4.0), which permits the noncommercial replication and distribution of the article with the strict proviso that no changes or edits are made and the original work is properly cited (including links to both the formal publication through the relevant DOI and the license). See: https://creativecommons.org/licenses/by-nc-nd/4.0/.

\section{References}

1. Cribier A, Eltchaninoff H, Bash A, et al. Percutaneous transcatheter implantation of an aortic valve prosthesis for calcific aortic stenosis: first human case description. Circulation 2002;106:3006-8.

2. Leon MB, Smith CR, Mack M, et al. Transcatheter aortic-valve implantation for aortic stenosis in patients who cannot undergo surgery. N Engl J Med 2010;363:1597-607.

3. Smith CR, Leon MB, Mack MJ, et al. Transcatheter versus surgical aortic-valve replacement in high-risk patients. $\mathrm{N}$ Engl J Med 2011;364:2187-98.

4. Reardon MJ, Van Mieghem NM, Popma JJ, et al. Surgical or Transcatheter Aortic-Valve Replacement in Intermediate-Risk Patients. N Engl J Med 2017;376:1321-31.

5. Popma JJ, Deeb GM, Yakubov SJ, et al. Transcatheter Aortic-Valve Replacement with a Self-Expanding Valve in Low-Risk Patients. N Engl J Med 2019;380:1706-15.

6. Cubero-Gallego H, Dam C, Meca J, et al. Transcatheter aortic valve replacement (TAVR): expanding indications to low-risk patients. Ann Transl Med 2020;8:960.

7. Van Belle E, Vincent F, Labreuche J, et al. BalloonExpandable Versus Self-Expanding Transcatheter Aortic Valve Replacement: A Propensity-Matched Comparison From the FRANCE-TAVI Registry. Circulation 2020;141:243-59.

8. Ihdayhid AR, Michail M, McCormick L, et al. Complicated Interaction Between Balloon Expandable Sheath and Self-Expanding Aortic Bioprosthesis. JACC Cardiovasc Interv 2020;13:e11-3.

9. Deharo P, Bisson A, Herbert J, et al. Impact of Sapien 3 Balloon-Expandable Versus Evolut R Self-Expandable Transcatheter Aortic Valve Implantation in Patients With Aortic Stenosis: Data From a Nationwide Analysis. Circulation 2020;141:260-8.

10. Hu P, Liu XB, Liang J, et al. A hospital-based survey of patients with severe valvular heart disease in China. Int J Cardiol 2017;231:244-7.

11. Jilaihawi H, Wu Y, Yang Y, et al. Morphological characteristics of severe aortic stenosis in China: imaging corelab observations from the first Chinese transcatheter aortic valve trial. Catheter Cardiovasc Interv 2015;85 Suppl 1:752-61.

12. Li Y, Wei X, Zhao Z, et al. Prevalence and Complications of Bicuspid Aortic Valve in Chinese According to Echocardiographic Database. Am J Cardiol 2017;120:287-91.

13. Pan $W$, Zhou $\mathrm{D}$, Cheng L, et al. Aortic regurgitation is more prevalent than aortic stenosis in Chinese elderly population: Implications for transcatheter aortic valve replacement. Int J Cardiol 2015;201:547-8.

14. Binder RK, Rodés-Cabau J, Wood DA, et al. Transcatheter aortic valve replacement with the SAPIEN 3: a new balloon-expandable transcatheter heart valve. JACC Cardiovasc Interv 2013;6:293-300.

15. Liao YB, Zhao ZG, Wei X, et al. Transcatheter aortic valve implantation with the self-expandable venus A-Valve and CoreValve devices: Preliminary Experiences in China. Catheter Cardiovasc Interv 2017;89:528-33.

16. Zhou D, Pan W, Wang J, et al. VitaFlow ${ }^{\mathrm{TM}}$ transcatheter valve system in the treatment of severe aortic stenosis: One-year results of a multicenter study. Catheter Cardiovasc Interv 2020;95:332-8.

17. Mosleh W, Amer MR, Joshi S, et al. Comparative Outcomes of Balloon-Expandable S3 Versus SelfExpanding Evolut Bioprostheses for Transcatheter Aortic Valve Implantation. Am J Cardiol 2019;124:1621-9.

18. Bavaria JE, Desai ND, Cheung A, et al. The St Jude Medical Trifecta aortic pericardial valve: results from a global, multicenter, prospective clinical study. J Thorac Cardiovasc Surg 2014;147:590-7.

19. Makkar RR, Yoon SH, Leon MB, et al. Association Between Transcatheter Aortic Valve Replacement for Bicuspid vs Tricuspid Aortic Stenosis and Mortality or Stroke. JAMA 2019;321:2193-2202.

20. Mauri V, Kim WK, Abumayyaleh M, et al. Short-Term Outcome and Hemodynamic Performance of NextGeneration Self-Expanding Versus Balloon-Expandable Transcatheter Aortic Valves in Patients With Small Aortic Annulus: A Multicenter Propensity-Matched Comparison. Circ Cardiovasc Interv 2017;10:e005013.

21. Husser O, Kim WK, Pellegrini C, et al. Multicenter Comparison of Novel Self-Expanding Versus BalloonExpandable Transcatheter Heart Valves. JACC Cardiovasc 
Interv 2017;10:2078-87.

22. Lee YJ, Lee SJ, Hong SJ, et al. Comparison of Transcatheter Aortic Valve Replacement between SelfExpanding versus Balloon-Expandable Valves in Patients with Small Aortic Annulus. Korean Circ J 202 1;51:222-31.

23. Mack MJ, Leon MB, Smith CR, et al. 5-year outcomes of transcatheter aortic valve replacement or surgical aortic valve replacement for high surgical risk patients with aortic stenosis (PARTNER 1): a randomised controlled trial. Lancet 2015;385:2477-84.

24. Athappan G, Patvardhan E, Tuzcu EM, et al. Incidence, predictors, and outcomes of aortic regurgitation after transcatheter aortic valve replacement: meta-analysis and systematic review of literature. J Am Coll Cardiol 2013;61:1585-95.

25. Ando T, Briasoulis A, Telila T, et al. Does mild paravalvular regurgitation post transcatheter aortic valve implantation affect survival? A meta-analysis. Catheter Cardiovasc Interv 2018;91:135-47.

Cite this article as: Ou-Yang WB, Wang W, Dong J, Xie YQ, Wan JY, Yue ZQ, Wang SZ, Meng H, Wang X, Xu DH, Zhang FW, Dong J, Pan XB, Zhang GJ. Propensity-matched comparison of balloon-expandable and self-expanding valves for transcatheter aortic valve replacement in a Chinese population. Ann Transl Med 2022;10(2):97. doi: 10.21037/atm-21-6637
26. Kim WK, Bhumimuang K, Renker M, et al. Determinants of paravalvular leakage following transcatheter aortic valve replacement in patients with bicuspid and tricuspid aortic stenosis. Eur Heart J Cardiovasc Imaging 2021. [Epub ahead of print].

27. Sammour Y, Krishnaswamy A, Kumar A, et al. Incidence, Predictors, and Implications of Permanent Pacemaker Requirement After Transcatheter Aortic Valve Replacement. JACC Cardiovasc Interv 2021;14:115-34.

28. van Rosendael PJ, Delgado V, Bax JJ. Pacemaker implantation rate after transcatheter aortic valve implantation with early and new-generation devices: a systematic review. Eur Heart J 2018;39:2003-13.

29. Osman M, Ghaffar YA, Saleem M, et al. Meta-Analysis Comparing Transcatheter Aortic Valve Implantation With Balloon Versus Self-Expandable Valves. Am J Cardiol 2019;124:1252-6.

(English Language Editor: A. Kassem) 